\title{
Las políticas de egreso desde experiencias situadas. Producción de diagnósticos, aprendizajes y desafíos
}

\section{Reflections on difficulties, learnings, and challenges on a graduation enhancement programme}

\section{Ornela Boix}

ornelaboix@gmail.com

CONICET - Centro Interdisciplinario de Metodología de las Ciencias Sociales/Instituto de Investigaciones en Humanidades y Ciencias Sociales (CONICETUniversidad Nacional de La Plata), Argentina

\section{Victoria D'Amico}

victoriadamico@gmail.com Instituto de Investigaciones en Humanidades y Ciencias Sociales (CONICET-Universidad Nacional La Plata), Argentina

\section{Juliana Frassa}

jfrassa@yahoo.com.ar Instituto de Investigaciones en Humanidades y Ciencias Sociales (CONICET-Universidad Nacional de La Plata), Argentina Instituto de Administración y Ciencias Sociales, Universidad Nacional Arturo Jauretche, Argentina

\section{Magdalena Lemus}

magdalenalemus.2@gmail.com

CONICET - Centro Interdisciplinario de

Metodología de las Ciencias Sociales/Instituto de Investigaciones en Humanidades y Ciencias Sociales (CONICET-Universidad Nacional de La Plata), Argentina

\section{Recepción: 03 Julio 2021}

Aprobación: 01 Agosto 2021

Publicación: 01 Diciembre 2021

Cita sugerida: Boix, O., D’Amico, V., Frassa, J. y Lemus, M. (2021). Las políticas de egreso desde experiencias situadas. Producción de diagnósticos, aprendizajes y desafíos. Cuestiones de Sociología, 25, e129. https://doi.org/10.24215/23468904e129
Resumen: Este artículo reconstruye y analiza la implementación del Taller de Tesinas y la Tutoría de Egreso, dos políticas de promoción del egreso implementadas para las carreras del Departamento de Sociología de la Facultad de Humanidades y Ciencias de la Educación (UNLP). Luego de situar dichas políticas desde la perspectiva de la educación superior como derecho, abordamos de qué manera permitieron diagnosticar características, obstáculos y dificultades para el egreso, y con este nuevo conocimiento, intervenir sobre las trayectorias estudiantiles. A la vez, sostenemos que un efecto expandido de estas políticas fue ampliar las estrategias desde las cuales producir política académica, lo que nos permitióconstruir nuevas perspectivas sobre el perfil de estudiantes que habitan nuestras carreras; el modo en que está funcionando en la práctica el plan de estudios y la compleja relación entre los soportes institucionales que acompañan la elaboración de las tesinas, las incumbencias profesionales de la Licenciatura y los formatos de acreditación de saberes vigentes. De este modo, el artículo pone en valor un abordaje multidimensional y situado del egreso, que enfatiza la dimensión académico-institucional. En esa clave, y a modo de conclusión, se elaboran propuestas que podrían favorecer el egreso en nuestras carreras y se reflexiona sobre la relación entre diagnósticos, políticas y evaluación, remarcando la importancia de las intervenciones específicas y de modos de evaluación más reflexivos y ajustados al conocimiento obtenido en la implementación de las políticas.

Palabras clave: Educación universitaria, Inclusión, Trayectorias educativas, Políticas de promoción del egreso.

Abstract: Since 2018 the National University of La Plata (UNLP) has engaged vigorously in the development of programmes to improve academic performance and increase graduation rates. In this paper we analyze the overall results of two initiatives, Taller de Tesinas and Tutoría de Egreso to date, put in place by the Department of Sociology, School of Educational Science and Humanities $(U N L P)$ in order to tackle student withdrawal and reduce graduation obstacles. First we describe each initiative, their main features and actions taken. Then, we evaluate the potential of both initiatives to produce meaningful data that allows us to identify and understand student withdrawal and difficulties regarding graduation, as well as planning more effective strategies to address these issues. While Taller de Tesinas and 
Tutoria de Egreso were mainly focused in helping students who had disfavorable contexts to take final exams or writing their bachelor thesis, they also had unexpected and beneficial effects on higher education policy making at the faculty level. Both initiatives allowed us to deepen our knowledge on current teaching and learning practices in local Sociology programmes, and to identify Sociology students profiles, including their interests, their educational trajectories and their job aspirations. The evaluation of both initiatives also highlighted the importance of certain institutional support policies to help students deal with obstacles they might face at the final stages and, in particular, to successfully finish their bachelor thesis. Finally, we identify challenges ahead in order to keep improving graduation rates and preventing student withdrawal. Moreover, we find that stress that diagnosis, strategies and evaluation are key to policy making and need to be considered within specific contexts and institutional framework.

Keywords: Undergraduate education, Inclusion, Educational trajectories, Graduation, enhancement programmes.

\section{Introducción: El egreso desde la perspectiva de la educación superior como derecho}

Este artículo se propone reconstruir y analizar las experiencias institucionales de implementación de dos políticas tendientes a la promoción del egreso en la Licenciatura y el Profesorado en Sociología de la Facultad de Humanidades y Ciencias de la Educación (FaHCE) de la Universidad Nacional de La Plata (UNLP): el Taller de Tesinas y la Tutoría de Egreso. La presente sistematización se basa en nuestra experiencia como docentes y tutoras de estas estrategias institucionales diseñadas para promover la graduación, las cuales nos han puesto en contacto cotidiano y nos han invitado a reflexionar sobre el problema de la terminalidad en la universidad, en general, y en las carreras del Departamento de Sociología, en particular, desde nuestro compromiso con dichas políticas. Aunque el Taller y la Tutoría han surgido en momentos diferentes y tienen objetivos diversos en el marco de las políticas de promoción del egreso, comparten diagnósticos, preocupaciones, perspectivas sobre la inclusión universitaria y modos de acción comunes.

Desarrollaremos en detalle cada una de estas estrategias en las siguientes secciones del artículo, pero es necesario adelantar ahora algunos de sus rasgos centrales. El Taller de Tesinas, por un lado, surge en 2013 en la órbita del Departamento de Sociología y su Junta Asesora, en las que convergen representantes de estudiantes, graduados/as y profesores/as, tres de los cuatro claustros que conforman el co-gobierno universitario. El Taller es extracurricular en tanto no forma parte del Plan de Estudios de la Licenciatura y tiene su origen en la detección de dificultades en el proceso de producción del trabajo final o tesina. Se orienta a acompañar a los/as estudiantes en la formulación de un problema de investigación en términos sociológicos y a la elaboración de un proyecto de tesina. Por otro lado, la Tutoría de Egreso comienza sus acciones en 2018 en el marco del Programa de Rendimiento Académico (PRAE) de la 
UNLP, creado ese año con el objetivo de alentar la permanencia en las carreras de grado y favorecer el egreso. ${ }^{1}$ Una de las características del PRAE es que, si bien hay estrategias de acción compartidas para todas las Unidades Académicas, se propicia la implementación de acciones específicas sobre la base de los desafíos diagnosticados en cada carrera. Una de esas estrategias es el "Acompañamiento para el egreso", dentro de la cual se enmarcan las intervenciones que viene llevando adelante la Tutoría de egreso de nuestro Departamento. Estas apuntan a acompañar a estudiantes en su tramo final de las dos carreras de Sociología (Licenciatura y Profesorado) y contemplan acciones muy variadas, desde brindar información y facilitar el contacto con cátedras hasta asesorar en la preparación de finales y diseñar, junto con las cátedras, modalidades de acreditación ajustadas a situaciones específicas.

Ahora bien, estas estrategias específicas no se pueden comprender acabadamente en sus objetivos y horizontes si no las enmarcamos en un contexto académico y político de más amplio alcance, que implica entender la educación superior como derecho. En la última década, ha crecido en América Latina una perspectiva que, contra la avanzada neoliberal de los años noventa, reconoce la educación superior como bien colectivo, como permiten ver los acuerdos sucesivos construidos en la Conferencia Regional de Educación Superior (CRES). ${ }^{2}$ Como sostienen Del Valle y Suasnábar (2018), este movimiento implicó por un lado "una resignificación de las nociones de acceso, expansión y democratización y, por otro, rediseñar y repensar los dispositivos y capacidades estatales para garantizar esa ampliación de derechos" (2018, p. 26). Este clima regional, que reconceptualiza la problemática educativa, fue reforzado a nivel nacional en el año 2015, cuando se modificó la Ley de Educación Superior para garantizar la gratuidad de la educación en las universidades públicas. ${ }^{3}$ El derecho a la universidad y la explicitación de su gratuidad modificó la lente a partir de la cual leer los desafíos institucionales para el acceso, la permanencia y el egreso.

Con una orientación convergente a estas transformaciones, en los últimos años la UNLP ha profundizado la implementación de políticas orientadas a la inclusión con calidad. A las políticas de seguimiento y apoyo de las trayectorias estudiantiles para lograr crecientes niveles de retención, se fueron sumando de manera progresiva desde 2010 programas de egreso que prestan atención a diversos obstáculos detectables en el tramo final de las carreras de grado. Se planteó, así, contribuir a la implementación de estrategias específicas para incrementar los niveles de graduación efectiva de estudiantes, cuyas trayectorias permanecían inconclusas, aun cuando se encontraban en un estado muy avanzado o final de desarrollo. Esto implicó el despliegue de modificaciones curriculares, programas de tutorías, cursadas especiales y estrategias de promoción del egreso, que constituyeron un nuevo piso de diagnóstico y de política académica sobre el cual, a partir de 2018, se dio un nuevo impulso con el PRAE.

En ese marco, la problemática de la graduación implica tratar con personas que, con un alto avance en sus estudios universitarios (más del $80 \%$ de la carrera aprobada, y en algunos casos, incluso más del 95\%), no logran finalizarlos.. Nuestra perspectiva política nos lleva a entender que, si bien el tránsito por las instituciones universitarias es de por sí enriquecedor para la socialización y formación ciudadana, es responsabilidad de las instituciones estatales promover 
la finalización de los estudios y su acreditación como parte del acceso efectivo al derecho a la educación superior. En este sentido, si el primer momento de la democratización de los estudios superiores se orienta, como plantea Rinesi (2015), a su universalización -a través de políticas orientadas a generar condiciones de acceso igualitarias para toda la población-, acordamos con que un segundo momento requiere que las instituciones estatales sean garantes también de generar las condiciones para la finalización. En otras palabras, se trata de asegurar que las trayectorias estudiantiles no se vean truncadas, al menos en principio, por condiciones académicas propias de la dinámica institucional, que en muchos casos pueden operar con sentidos expulsivos sobre una parte de la población estudiantil.

Desde este posicionamiento político, estas estrategias requirieron de imaginación para producir intervenciones no curriculares que pudieran institucionalizarse. Con su modo de hacer, tanto el Taller de Tesinas en primera instancia, como la Tutoría de Egreso luego, permitieron que el Departamento de Sociología recuperara información, experiencias y relatos que se convirtieron en insumos para construir nuevos diagnósticos acerca de las variables que se encuentran operando en la interrupción de trayectorias estudiantiles muy avanzadas. Dichas variables son diversas: desde circunstancias biográficas particulares (de tipo laboral, familiar o de radicación residencial) que dificultan la terminalidad y/o provocan la pérdida de contacto cotidiano con la facultad, lo que favorece el abandono; hasta condicionantes académicos vinculados tanto a los miedos y exigencias de los/as estudiantes en torno a exámenes y trabajos finales como a los efectos de regímenes de acreditación más flexibles que, al tiempo que promueven la autonomía y la creatividad, pueden conducir a la dilatación en la presentación de trabajos y otras actividades necesarias para acreditar las materias. Específicamente en el caso de la tesina, la instancia más adeudada por los/as estudiantes en el tramo final de la Licenciatura y el desafío más grande para el egreso en el Departamento de Sociología, ${ }^{4}$ se anudan la dificultad de no haber podido avanzar en la elección de un tema, área o problema de interés en el transcurso del recorrido por las asignaturas y la persistencia, más general, de imaginarios sobre este trabajo final de grado que responden a otros momentos históricos de la carrera, e inclusive a planes de estudios previos, en los que predominaba una orientación hacia la formación en investigación.

La información recuperada a partir de estas políticas planteó el desafío de revitalizar el plan de estudios existente, no necesariamente a partir de su modificación (proceso institucional de mediana duración, por los actores y debates involucrados) sino, en el "mientras tanto", buscando producir innovaciones de inmediata implementación que permitieran reconocer y dar lugar a una multiplicidad y heterogeneidad de trayectorias estudiantiles que transitan por nuestras carreras, que a lo largo del tiempo y de los cambios de contexto varían, y que impulsan a pensar- con ideas renovadas y flexibles- cómo se desarrolla la formación. Dispositivos de intervención y política académica fueron, entonces, produciendo un diálogo virtuoso que buscamos reponer en estas páginas.

Dicho diálogo está motorizado por algunas preguntas centrales. ¿Qué políticas implementa el departamento de Sociología, como parte de la FaHCE, para 
favorecer la graduación de sus estudiantes en la etapa de culminación de la carrera? ¿Cuáles son las características de la intervención pedagógica que se realiza en el Taller de tesinas? ¿Qué acciones de acompañamiento y seguimiento realiza la Tutoría de Egreso? ¿Cómo ambas políticas han entrado en contacto y se retroalimentan? ¿De qué manera considerar el impacto de estas propuestas? ¿Cómo interpelan a los indicadores disponibles para medir la actividad académica y el egreso? ¿Cuáles son los principales desafíos que presentan ambas líneas de intervención? Y, finalmente, ¿cómo estos dispositivos habilitan reflexiones sobre la dinámica de los planes de estudios de las carreras, su relación con la terminalidad y con las incumbencias profesionales? De este modo, apostamos tanto a compartir los rasgos de experiencias institucionales concretas como a contribuir en un debate abierto sobre la terminalidad en la universidad y el papel que allí juegan las políticas académicas.

\section{Políticas institucionales: diagnósticos situados, prácticas y sinergias}

\subsection{Taller de Tesinas}

El Taller de Tesinas es un espacio extracurricular creado por el Departamento de Sociología en el año 2013. En sus inicios se propuso como política orientada a la graduación de estudiantes de licenciatura que únicamente adeudaban la tesina final para la obtención del título de grado. Por entonces, la población destinataria estaba conformada por estudiantes que habían cursado la totalidad de las materias de la carrera, no contaban con avances en la tesina y habían perdido el vínculo con la facultad, considerando que su última actividad académica (rendido su último final o cursado sus últimas materias) databa de tres años antes. De esa manera, quienes participaban del Taller eran, inicialmente, personas que necesitaban reconstruir el lazo con la institución, porque habían perdido la cotidianeidad de circular por ella y no habían quedado vinculadas a otros marcos colectivos (tales como cátedras, proyectos de investigación o proyectos de extensión), que operan muchas veces como condición de posibilidad para el desarrollo de los trabajos finales de carrera. A medida que el Taller de Tesinas se fue consolidando como espacio de acompañamiento de las trayectorias educativas en su tramo final, la matrícula de estudiantes fue aumentando, de modo que ya no sólo se incorporaban estudiantes que habían perdido el vínculo con la facultad sino, también, quienes cursaban el último año de la carrera. En este sentido, en los últimos años la matrícula del Taller se sostuvo entre los 20 y 30 estudiantes promedio, que se distribuyen en tres comisiones de trabajo.

Los objetivos del Taller han ido redefiniéndose a lo largo de su implementación. Podemos afirmar que el objetivo prioritario fue, en sus inicios, el de revincular a los/as estudiantes con la institución académica considerando que, para la producción de conocimiento, es recomendable enlazarse a otros sujetos individuales o colectivos (directores/as, compañeras/ os, docentes, cátedras, proyectos de investigación y de extensión) que habiliten un espacio colectivo de intercambio. En este sentido, el Taller propone una ruptura frente a una visión tradicional de la producción de conocimiento científico asociada a la figura individual del erudito/a que, a partir de sus propias 
reflexiones, alcanza un resultado original de investigación. Por el contrario, el Taller construye un encuentro colectivo de circulación de saberes, habilidades y experiencias que, en su sinergia, colabora tanto con la revinculación académica como con la formulación del proyecto de tesina de cada estudiante. En este sentido, el Taller se dispone, por un lado, como vínculo entre los/as estudiantes y, por otro lado, como espacio a partir del cual establecer puentes con directores/ as de tesinas y/o proyectos de investigación y extensión que puedan actuar como marco de desarrollo de las tesinas.

Una vez iniciada esa revinculación entre pares, el Taller se propone una tarea de corte propiamente académico, orientada a trabajar en la formulación de un problema de investigación pertinente a la carrera. En este sentido, el Taller busca lograr como resultado la elaboración de un proyecto de tesina. A partir de allí, la segunda tarea del Taller es enlazar a esa persona y su propuesta de investigación con una dirección posible; es decir, acortar la distancia con la institución y entramar a esas personas en la red institucional necesaria para desarrollar esa última instancia requerida por el plan de estudios. Asimismo, aunque con otra urgencia, el Taller se propone acompañar a quienes ya se hallan en proceso de elaboración y redacción de sus tesinas y encuentran aquí la posibilidad de organizar su trabajo en torno a fechas de entrega y a la posibilidad de recibir comentarios de lectores pares. En este caso, el Taller también ha habilitado la posibilidad de colectivizar la experiencia de desarrollo de la tesina.

En términos de la intervención pedagógica, el Taller se estructura en una serie de encuentros quincenales, desarrollados a lo largo de un cuatrimestre, en los que los/as estudiantes elaboran cinco guías de trabajo procesuales y progresivas que los/as orientan hacia el diseño de sus proyectos de tesina. De modo específico, las actividades desarrolladas en el marco del Taller se refieren a: conocer los requisitos, formatos y estilo que supone una tesina de grado, leer y comentar una tesina, realizar búsquedas bibliográficas, construir preguntas y objetivos de investigación, revisar nociones básicas de metodología de la investigación social y, finalmente, construir un proyecto de tesina. La metodología de trabajo combina presentaciones por parte de los/as estudiantes de sus avances y entrega y devoluciones de guías de trabajo. Cabe destacar que la principal dinámica del Taller consiste en el proceso de retroalimentación entre la totalidad de estudiantes a partir de ejercicios de comentarios y correcciones cruzadas: todos/ as leen a otro/a y son leídos por otro/a.

De acuerdo con los distintos objetivos que se propuso el Taller podemos identificar como principales resultados:

-Lograr el acercamiento y re-vinculación institucional de una numerosa cantidad de estudiantes que habían "desenganchado" sus trayectorias educativas de la facultad y que, en principio, ahora contaban con un espacio pensado para la elaboración de sus tesinas;

-Discutir sobre los alcances y requisitos básicos de la tesina, ayudando a deconstruir ciertos imaginarios establecidos en torno a su realización. Esto implicó una "descompresión" de la carga que suponía la tesina para gran parte de la población estudiantil que, muchas veces, paralizaba por ello su elaboración;

-Promover el ejercicio de la escritura académica;

-Favorecer el vínculo con directores/as de tesinas y reconstruir el espacio colectivo de reflexión y acompañamiento en el tramo final de la carrera que 
supone el desarrollo de la tesina. Más allá de la disparidad de temáticas abordadas, el hecho de compartir el mismo proceso promueve el intercambio y la contención mutua entre quienes asisten.

Además de intervenir sobre las situaciones específicas de los/as estudiantes que se acercaron al Taller, la experiencia anual que se fue replicando nos permitió construir un diagnóstico más preciso acerca de los motivos por los cuales las personas, encontrándose en una situación tan cercana a la graduación, no lograban culminar el proceso de acreditación de sus estudios universitarios. Así, además de los acontecimientos biográficos que permitían explicar algunos de esos casos (dejar la ciudad de La Plata para retornar a la ciudad de origen, acceso a un trabajo que imposibilitaba dedicar horas al proceso de la tesina, maternidad/paternidad) comenzaron a resonar sobre el espacio interrogantes acerca de por qué esas personas no habían logrado realizar la tesina en el transcurso mismo de la carrera. Es decir, más allá de que sobre esas situaciones particulares teníamos que intervenir proponiendo espacios ad hoc que volvieran a generar condiciones de posibilidad para la finalización, la experiencia nos llevó a construir un diagnóstico colectivo que permitió identificar algunas de las variables académicas estructurales vinculadas al trayecto formativo de la carrera que exceden las decisiones biográficas.

En el desarrollo del Taller se evidenció la existencia de una masa crítica de casos que comparte la dificultad para realizar la tesina en tanto esta aparece en sus representaciones como una "caja negra" que no se sabe cómo funciona. En este sentido, el diagnóstico sobre el problema de la tesina ya no queda anclado a un asunto meramente individual. La recurrencia nos invitó a revisar qué sucede con las condiciones institucionales y académicas a partir de las cuales una persona pueda producir ese objeto llamado tesina en continuidad con su recorrido por las materias del plan de estudios. Ya no se trata entonces de intervenir sobre casos particulares que se "alejan" de la trayectoria esperada y de los plazos de formación previstos por el Plan de estudios sino de repensar concepciones y modelos de graduación, y rediseñar estrategias, procedimientos y articulaciones interinstitucionales para mejorar los indicadores de egreso. En este sentido, la elaboración de la tesina en el marco del Taller se volvió una instancia potente para revisar problemáticas, tensiones y desarticulaciones preexistentes.

\subsection{Tutoria de Egreso}

Los lineamientos generales del plan estratégico de la UNLP y del PRAE han llevado a la implementación de tutorías de egreso en las distintas facultades. Desde la FaHCE hemos comenzado en 2018 a desarrollar tutorías de egreso que promueven la revinculación con la vida académica y la reanudación de la trayectoria de estudiantes que, por diversos motivos, se han alejado de las carreras en las que ya habían realizado un avance significativo. Con este objetivo como guía, las tutorías de egreso han desarrollado diagnósticos específicos para cada carrera, así como han delineado intervenciones para promover la acreditación de cursadas y finales del último tramo de la carrera. Específicamente en el Departamento de Sociología, el trabajo se ha concentrado en el acompañamiento para la acreditación de los talleres, las materias optativas y las materias obligatorias 
de los últimos dos años del plan sugerido y, en el caso particular de la Licenciatura, en una serie de estrategias para facilitar la producción de la tesina o trabajo final.

Desde el comienzo de la Tutoría de Egreso en el año 2018 venimos realizando diagnósticos sobre la base del análisis de distintos conjuntos de casos extraídos de las nóminas de estudiantes de nuestras dos carreras, derivadas de los reportes del Centro Superior para el Procesamiento de la Información (CeSPI) de la UNLP. Estos datos nos han permitido contactar a estudiantes de forma personalizada. A su vez, desde los inicios del programa hasta la actualidad, se trabaja con casos que llegan por otras vías, como derivaciones realizadas por el Departamento de Sociología o por docentes, por bola de nieve dentro del propio programa, etc. Junto a esto, se han explorado modalidades de convocatoria a través de campañas de difusión por la web y las redes sociales del Departamento y de los distintos claustros.

Durante los tres años que lleva en marcha, la Tutoría de egreso identificó distintas problemáticas en el tramo final, entre ellas, la dificultad para rendir exámenes finales de las últimas materias obligatorias y la dilatación de la entrega de trabajos finales (monografías, proyectos de investigación, informes) de materias optativas y talleres de investigación del denominado ciclo superior o especializado. Estas situaciones, que se manifiestan por igual en trayectos de la Licenciatura y el Profesorado, suelen obedecer a un alejamiento de la vida estudiantil, que tiene en la base motivos residenciales, laborales, familiares (entre los que incluimos tareas de cuidado), a la marcada postergación para rendir exámenes finales como también a los efectos de propuestas de acreditación de las materias del tramo final, especialmente en optativas de tipo C, D o E, Teoría Social Contemporánea B y talleres de investigación, ${ }^{5}$ las cuales suelen aprobarse con trabajos escritos (informes, monografías, ensayos, proyectos de investigación) con consignas y tiempos de realización menos estructurados que los parciales y exámenes finales con los cuales los/as estudiantes están familiarizados/as desde el inicio de la carrera. Frente a estos problemas, se trabajó con algunas pautas básicas: 1) establecer contacto con distintas cátedras para acordar un plan personalizado para la realización de trabajos y/o exámenes finales según las modalidades contempladas en el Régimen de Enseñanza y Promoción; 2) matricular a los/as estudiantes en las aulas virtuales del Campus FaHCE para facilitar el acceso al material de estudio; 3) resolver distintos problemas administrativos en la historia académica (analítico) de los/as estudiantes, en conjunto con el equipo de gestión del Departamento de Sociología; 4) asesorar en la planificación de los procesos de cursada y presentación en exámenes finales. A medida que se avanzaba en el conocimiento de las trayectorias estudiantiles y se pudo contar con datos más precisos y desagregados, la Tutoría también pudo realizar otras intervenciones: organizar cohortes específicas del Taller de Tesinas (por ejemplo, de estudiantes trabajadores/as en el Estado) y, con el apoyo y el trabajo de las cátedras, armar cursadas intensivas para la acreditación de determinadas materias del tramo final.

Sin embargo, como ya adelantamos, la dificultad preponderante para la graduación en el Departamento de Sociología es la tesina (Plan 2001, Ingreso 2003) o trabajo final (Plan 1991), considerada la última materia de la Licenciatura. Como se planteó en la sección anterior, el Taller de Tesinas nos permitió contar con información desagregada acerca de diversos motivos 
que coinciden en obstaculizar su realización: la combinación compleja de características de este producto escrito, formatos institucionales, costumbres e imaginarios. La tesina presenta características diferentes del resto de las producciones escritas que se elaboran a lo largo de la carrera (parciales, monografías, proyectos de investigación, informes), se realiza individualmente y, en general, luego de terminadas las cursadas y no necesariamente en vinculación con espacios curriculares y/o proyectos de investigación colectivos. Por lo tanto, las intervenciones para contribuir a la realización y finalización de la tesina implican también esfuerzos distintos de los del resto de las materias y revisten, tanto en quienes egresan como en quienes no, así como en sus directores/as y codirectores/as, diversos desafíos

Desde su origen en 2018, la Tutoría trabajó/a con una mayoría de estudiantes que debían sólo la tesina para su graduación y lo hizo a partir de: 1) establecer contacto entre el/la estudiante y posibles directores/as y co-directores/as de tesina, de acuerdo con las definiciones y avances temático-metodológicos de cada caso; 2) seguir los procesos de realización ypresentación de tesinas en el Departamento; 3) poner en contacto al/la estudiante con el Taller de Tesinas en los casos en que no existe un tema o avances definidos para trabajar. Este contacto no implica meramente una derivación, sino un compromiso entre la Tutoría y el Taller para realizar un seguimiento conjunto de los casos. Este trabajo común con las docentes del Taller continuó y se amplió con el correr del tiempo. Así, ambos espacios están coordinados a la hora de realizar la convocatoria anual al Taller de Tesinas o para planificar actividades relacionadas con el tema. Esta labor mancomunada permitió darle integralidad al problema de la tesina en el Departamento, a la vez que afinar los diagnósticos sobre cuáles son las dificultades y sus posibles soluciones.

Con el objetivo de sistematizar e institucionalizar las experiencias que se venían llevando a cabo alrededor de la tesina y hacer que las herramientas brindadas por la Tutoría de manera personalizada lleguen a más estudiantes, desarrollamos acciones específicas, en articulación con la Dirección del Departamento y los/as representantes de los claustros de estudiantes, graduados/ as y profesores/as en la Junta Asesora Departamental:

- Jornada de tesinas: Se realizó en junio de 2019 y surgió de un conjunto de diagnósticos obtenidos a partir del contacto directo con estudiantes en el tramo final en el trabajo diario de la Tutoría; de la lectura de las respuestas a las encuestas abiertas hechas entre quienes se inscribieron al Taller de Tesinas entre los años 2014-2018 y su presentación y debate en una reunión del banco de directores y evaluadores realizada en noviembre de 2018; de diagnósticos de la práctica docente en el Taller de Tesinas y, finalmente, de reflexiones previas de larga data ancladas en el Departamento de Sociología. Durante esa jornada los distintos claustros compartieron sus experiencias y miradas respecto de las tesinas. El espacio buscó poner de relieve las dificultades manifestadas por los distintos actores involucrados en el proceso de producción de una tesina y contribuyó a crear perspectivas compartidas acerca de las características y alcance que debe tener.

- Revisión de las "Pautas generales para la elaboración del trabajo final (plan 1991) o tesina (plan 2003) de la licenciatura en Sociología": Articulada desde la Junta Asesora Departamental con la participación de los claustros 
que la componen, esta tarea fue realizada durante 2019 y 2020 buscando sumar dinamismo y claridad a los lineamientos ya existentes, bajo un formato de pregunta-respuesta que considera algunos de las dudas usuales que los/as estudiantes han manifestado en su conversación con las tutoras. Esta iniciativa también buscó poner de relieve el amplio espectro de modalidades de tesina que se pueden llevar a cabo, así como la variedad de temáticas que se pueden abordar. De esta manera, se intentó contribuir a ampliar los imaginarios sobre la tesina, a la vez que ajustarlos a las incumbencias profesionales que la carrera tiene efectivamente y que se encuentran en el horizonte profesional de estudiantes y graduados/as.

- Recursero Bibliográfico para la realización de tesinas/trabajo final en Sociología: Se trata de un espacio virtual alojado en el campus de la FaHCE que fue lanzado en 2020 y que funciona como repositorio de bibliografía de apoyo metodológico, pensado específicamente para el tránsito por la tesina, que complementa los contenidos tratados en las materias metodológicas y busca, especialmente, acompañar a los/as estudiantes, directores/as y codirectores/as durante la realización de la tesina.

-Construcción de datos sobre problemáticas asociadas al egreso que se ubican en la intersección de dimensiones académicas con socioeconómicas, laborales y de género. Creemos que identificar de qué modo participan estas dimensiones en las problemáticas de egreso nos va a brindar información valiosa para el diseño de futuras intervenciones. De manera preliminar, podemos señalar que el trabajo con la población involucrada confirma el impacto regresivo de las dinámicas residenciales, laborales y familiares en el egreso.

\section{Aprendizajes a partir del diálogo entre políticas}

Como ocurre con distintas políticas públicas, su implementación y apropiación por parte de las personas e instituciones destinatarias suelen desbordar los cálculos y planificaciones previas, y producir articulaciones variadas entre la política y sus destinatarios/as, al tiempo que enriquecen nuevos procesos de diseño y puesta en práctica. En el caso de políticas como el Taller de Tesinas y la Tutoría de Egreso, podemos identificar resultados concretos en distintas dimensiones y también una suerte de "efecto expandido" inesperado. Entendemos que el diálogo y la práctica conjunta nos han permitido construir nuevas perspectivas sobre el perfil de estudiantes que habitan la carrera, y diagnósticos más ajustados sobre el modo en que está funcionando en la práctica en el plan de estudios y su relación con el mercado de trabajo realmente existente para los/as graduados/as, entre otras cuestiones.

A partir del trabajo conjunto entre el Taller de tesina y la Tutoría de Egreso, hemos identificado que las dificultades relacionadas con la tesina se inscriben en un amplio espectro: desde cuestiones de socialización académica, como desconocer cuándo y cómo contactar a un/a posible director/ay cuáles son sus incumbencias, hasta problemas referidos a la propia formulación de una pregunta de investigación, la elaboración de un diseño metodológico o la escritura académica. En el quehacer cotidiano hemos detectado que estas dificultades no afectan sólo a una porción específica del estudiantado "alejada" de la carrera, sino que son comunes en el tránsito final de la Licenciatura y tienen un carácter académico y organizacional. Algunas de estas cuestiones son, de hecho, objeto 
de las intervenciones diarias de la Tutoría de egreso, por ejemplo a través de la difusión de las Pautas generales para la elaboración del trabajo final (plan 1991) o tesina (plan 2003) y la comunicación del tipo de trabajo que se espera. Sumado a estas razones académicas e institucionales, debemos señalar la escasez de tiempo disponible para cursar y realizar la tesina en el caso de estudiantes que trabajan y/ o realizan tareas de cuidado.

En un plano subjetivo, relativo a los/as destinatarios/as centrales de estas políticas, las intervenciones realizadas han estimulado la revinculación con la carrera y con la Facultad de cientos de estudiantes, ya sea para retomar las instancias pendientes como para, en numerosos casos, alcanzar efectivamente la titulación. En esta línea, el paso de los/as estudiantes por la Tutoría y el Taller de Tesinas fue, en términos de su trayectoria educativa y de su experiencia subjetiva, un disparador a partir del cual reconectarse con el objetivo de finalizar la carrera, que por circunstancias biográficas y académicas hasta entonces había sido vivido como una imposibilidad. En este sentido, el trabajo colectivo, el acompañamiento pedagógico en la elaboración del proyecto, el intercambio con estudiantes y docentes y la revinculación con el proyecto institucional de formación son los aspectos más valorados por los/as estudiantes en relación con el Taller. En sintonía con esto, quienes se encuentran finalizando la carrera en el marco de la Tutoría de Egreso destacan el puntapié institucional, que la Facultad "haya ido a buscarlas/os", para la revinculación con la carrera y la reactivación de su trayectoria educativa, que en todos los casos aparecía como una deuda pendiente, algo que se anhelaba y a lo que era deseable darle un cierre. Sumado a esto, el abordaje desde una perspectiva académico-institucional ha contribuido a correr el foco de las dificultades para el egreso del plano individual y a visualizarla como una problemática que afecta a estudiantes en diversidad de condiciones, y que debe ser entendida no sólo desde las elecciones y acciones llevadas a cabo individualmente, sino también en relación con las características de la inserción profesional de los/as sociólogos/as, la demanda del mercado de trabajo, las transformaciones del perfil de estudiantes y egresados/as y las dinámicas institucionales de la carrera.

En línea con esta última idea, entender la relevancia de ir produciendo conjuntamente prácticas y diagnósticos se constituye como el principal aprendizaje de estas políticas. La reflexión sobre las experiencias a medida que iban transcurriendo reconfiguraron nuestras prácticas en dos sentidos. Por un lado, colaboraron a replantear otros universos posibles de destinatarios/as. Como dijimos previamente, el universo de estudiantes sobre el que intervenir se fue ampliando con el correr del tiempo, de modo que se apuntó a que al Taller llegaran no solamente personas que habían perdido lazos con la facultad, sino que desde la Tutoría también se fueron buscando estrategias para incorporar a quienes se encuentran rindiendo los últimos finales y proponerles que comenzaran a imaginar el proceso de elaboración de la tesina antes de desconectarse completamente de la facultad. Con el mismo espíritu, pensamos la Jornada de Tesinas, el Recursero y otras propuestas. De esta manera, comenzamos a anticiparnos a las situaciones que habían originado las primeras intervenciones.

Por otro lado, el diálogo entre ambas experiencias abrió la revisión sobre el modo concreto en que estaba operando el plan de estudios de la carrera en el tramo final. Un hallazgo en este sentido se encuentra en la gran cantidad 
de estudiantes que adeudan trabajos finales de materias optativas y talleres, los cuales, replanteando dinámicas de trabajo, podrían funcionar como antesala o primer acercamiento a una tesina que continuara ese esfuerzo ya iniciado. ${ }^{6}$ Evidencias de esta clase nos condujeron a trabajar sobre algunos instancias institucionales específicas: reflexionar sobreel rol de las materias optativas y talleres de los últimos años en relación con la tesina; revisar la articulación entre las materias del área metodológica y el área de las teorías sociales para analizar sus puntos de encuentro y distancias; y repensar de manera integral cómo las diferentes materias que conforman la totalidad del plan de estudios podrían, desde aportes e iniciativas pertinentes a sus objetivos específicos y a su lugar en la trayectoria curricular, ofrecer herramientas formativas que explícitamente se propongan abordar algún aspecto de la elaboración de la tesina. En este sentido, consideramos significativo que se volvió a visibilizar y a compartir la responsabilidad sobre la tesina como tarea colectiva de las cátedras.

Recuperadas en el plano institucional, estas revisiones han alimentado y movilizado intercambios interclaustros en la Junta Asesora Departamental respecto de cómo entender y qué esperar del tramo final de la Licenciatura y el Profesorado en Sociología y, en lo atinente a la tesina, relativizar imaginarios que no estaban en sintonía con el tipo de instancia formativa que promueve el Departamento. Además, como ya vimos, estos intercambios se han plasmado en acciones conjuntas, como por ejemplo, la Jornada de tesinas y la revisión de las "Pautas Generales para la Elaboración del trabajo final (plan 1991) o tesina (plan 2003)”. De esta manera, las experiencias transitadas han contribuido a la visibilización y sensibilización entre los diferentes claustros respecto de las dificultades que entraña la realización de la tesina y acerca de su significativo impacto en las tasas de egreso.

En síntesis, las experiencias del Taller de Tesinas y de la Tutoría de Egreso, articuladas entre sí y revisadas a la luz de su continuidad por el conjunto de actores que definen la política departamental, habilitaron la exploración crítica de la relación existente entre las incumbencias profesionales de la licenciatura en Sociología y los formatos de acreditación de saberes vigentes; la interpretación disímil de las pautas generales para la elaboración del trabajo final (plan 1991) o tesina (plan 2003); y el papel de los soportes institucionales para el acompañamiento del proceso de elaboración de las tesinas. Sus aportes fueron, entonces, más allá del hecho de favorecer la presentación de tesinas y así incrementar la cantidad de graduados/as de la carrera. Por las condiciones en que han sido llevadas adelante, permitieron componer un mapa más amplio en términos de identificar, visibilizar y abordar limitaciones institucionales que estaban interviniendo en el egreso.

\section{Desafíos pendientes}

El recorrido hecho hasta aquí deja abiertos algunos desafíos con miras a continuar impulsando el egreso de estudiantes. Respecto a la tesina, instancia final necesaria para el egreso de la Licenciatura en Sociología, se abre un interrogante clave: ¿de qué manera seguir instalando el interés y acercamiento a la tesina antes de que se transforme en un obstáculo para la graduación? Las experiencias analizadas nos permiten imaginar algunas intervenciones transversales que podrían consolidar la 
formación y anticiparse a algunos de los problemas mencionados anteriormente. Entre algunas acciones podemos señalar: incorporar el trabajo con tesinas en materias finales de la carrera (ya sea con la tarea concreta de trabajar proyectos, de proponer tesinas posibles respecto de los contenidos vistos; o con estrategias complementarias, por ejemplo, leer tesinas vinculadas al campo de saberes que la materia aporta); visibilizar espacios institucionales para impulsar o desarrollar la tesina (que ya están contemplados en los planes de estudios pero se encuentran fuera del imaginario estudiantil, tales como proyectos de investigación y/o de extensión, talleres y materias optativas), entre otras pistas a profundizar. Sería productivo también fortalecer el rol del Departamento a fin de formular puentes para las demandas que recibimos individualmente como docentes y no podemos contener, de manera de no dejar "a la deriva" a los/as estudiantes que escriben pidiendo acompañamiento en la dirección. Una apuesta de más amplio alcance, acorde con los cambios de mediana duración que ha promovido la política de egreso, implicaría familiarizar a los/as estudiantes con el proceso de tesina desde los primeros años de la carrera y fortalecer la dimensión pedagógica de la tesina entre la comunidad académica del Departamento.

En un nivel más general y amplio al que hemos venido apuntando a lo largo del artículo, se pone de manifiesto que el éxito de estas políticas no se puede calibrar sólo por indicadores cuantitativos que midan las tasas de egreso y su variación. Más bien, la riqueza de producir este tipo de experiencias consiste en que pueden colaborar a transformar cualitativamente las condiciones de todo un proceso formativo. De este modo, dispositivos ad hoc que no forman parte del plan de estudios -el Taller de Tesinas, la Tutoría de Egreso, pero también los actuales dispositivos existentes en el ingreso, como el Curso Introductorio- pueden activar discusiones acerca de los contenidos de materias, los modos de enseñanza y de evaluación que sí forman parte de la currícula, y transformar así prácticas sedimentadas en la cultura institucional que conforman una carrera. Al hacerlo, estas experiencias implican un compromiso con las políticas de democratización de la universidad en cuanto reponen información, conocimiento institucional, habilidades, "saber hacer" y competencias que están desigualmente distribuidas tanto en el ingreso (Barrena et al., 2020; Aréchaga et al., 2015) como en el tramo final de las carreras.

Es necesario, entonces, repensar la relación entre diagnósticos, políticas y evaluación de resultados para mostrar la potencia que pueden tener las prácticas de intervención cuando se reflexiona sobre ellas y revierten en insumos necesarios para la producción de diagnósticos mejor situados, que permiten refinar hipótesis de trabajo y formular intervenciones mejor adaptadas y receptivas a problemáticas específicas de la población estudiantil. Asimismo, esto plantea el desafío de construir indicadores de progreso y de evaluación de las políticas que permitan captar resultados que no son rápidamente traducibles a índices estandarizados.

Para terminar, consideramos que revisar la política académica desde estas experiencias situadas permite seguir apostando a los procesos de democratización de la educación superior a través de la institucionalización de prácticas que, con la participación comprometida de todos los claustros, confluya hacia un posicionamiento más reflexivo acerca de los procesos formativos que prevén 
las carreras, las prácticas pedagógicas que los organizan y el vínculo entre sus diferentes actores.

\section{Referencias}

Alzugaray, L., Cafiero, B., \& Varela, S. (2012). Análisis estadístico de los trabajos incluidos en el Volumen 2 de Tesinas: Licenciatura en Sociología (publicación digital). Cuestiones De sociología, 8. Recuperado de https://www.cuestionessociolo gia.fahce.unlp.edu.ar/article/view/CSn08a23

Aréchaga, A. J., Beliera, A., Ornela, B., Corsiglia Mura, L., Cueto Rúa, S., Di Piero, M. E., Galar, S., Gubilei, E., \& Henry, M. L. (2015). Puentes hacia la inclusión universitaria. Un análisis a partir de la experiencia en el Curso de Ingreso a las carreras de Sociología de la FaHCE-UNLP (2012 - 2015). Cuestiones de sociología, 13. Recuperado de https://www.cuestionessociologia.fahce.unlp.edu.a r/article/view/CSn13a08

Barrena, M.et al (2020). Pensar el ingreso y el primer año. Un ejercicio de reflexión sobre la experiencia docente en el curso introductorio a las carreras de Sociología - FaHCE/UNLP. Cuestiones de Sociología, 23, e108. https://doi.org/10.24215/2 $3468904 \mathrm{e} 108$

Cotignola, M., Legarralde, M., \& Margueliche, J. C. (2017). Las trayectorias universitarias de estudiantes de Sociología de la FaHCE. Un análisis desde los registros administrativos. Cuestiones De sociología, 17, e045. https://doi.org/10.2 $4215 / 23468904 \mathrm{e} 045$

Del Valle, D. y Suasnábar, C. (Coords.) (2018). Politica y tendencias de la educación superior a diez años de la CRES 2008. Buenos Aires: IEC-CONADU-CLACSOUNA.

Departamento de Sociología (2001). Licenciatura en Sociología. Plan de la carrera 2001 (modificaciones al plan 1991). Recuperado del sitio de internet de la UNLP, FaHCE, Departamento de Sociología: https://www.fahce.unlp.edu.ar/facultad/secretarias-y-prosecretarias/ academica/deptos/sociologia/carreras/licenciatura-en-sociologia

Departamento de Sociología (2001). Profesorado en Sociología. Plan de la carrera 2001. Recuperado del sitio de internet de la UNLP, FaHCE, Departamento de Sociología: https://www.fahce.unlp.edu.ar/facultad/secretarias-y-prosecretarias/ academica/deptos/sociologia/carreras/profesorado-en-sociologia

Departamento de Sociología (2021). Pautas para la elaboración del Trabajo Final (Plan 1991) o Tesina (Plan 2003) de la Licenciatura en Sociología. Recuperado del sitio de internet de la UNLP, FaHCE, Departamento de Sociología: https://www.fahce.unlp.edu.ar/facultad/secretarias-y-prosecretarias/ academica/deptos/sociologia/tramites/tramite-210818114916689258

Di Bello, M. E., Fernández Berdaguer, M. L. y Santos, J. (2011). Trayectorias educativas y laborales de los graduados de Sociología de la Universidad Nacional de La Plata. Cuestiones de Sociología, 7. Recuperado de https://www.cuestionessociologia.fah ce.unlp.edu.ar/article/view/CSn07a15

Giordana, P. B., Erbetta, M. C. y Manuele, M. E. (2003). Final de juego: El perfil de la Carrera de Sociología a través de los trabajos finales de sus egresados. Cuestiones de Sociologia, 1, 211-215. Recuperado de http://www.memoria.fahce.unlp.edu.ar/a rt_revistas/pr.3402/pr.3402.pdf

Rinesi, E. (2015). Filosofia (y) politica de la universidad. Los Polvorines: UNGS-IEC. Colección Educación, Serie Universidad. 


\section{Notas}

1 Como veremos más adelante, el PRAE se inscribe en las políticas de apoyo a las trayectorias estudiantiles que forman parte del Plan Estratégico de la UNLP 2018-2022. Sus acciones ponen el foco en distintos momentos de las trayectorias, al momento del ingreso, el tramo medio y el trayecto final de las distintas carreras, con el objetivo de desarrollar estrategias para incrementar la retención, la permanencia y el egreso en las distintas Unidades Académicas. Dentro del PRAE se contemplan tres tipos de estrategias: las tutorías de apoyo al rendimiento académico para estudiantes de primer año y de tramos medios; la creación de nuevas oportunidades para la aprobación de materias a través de cursadas intensivas o alternativas, y las políticas de acompañamiento para el egreso.

2 La Conferencia Regional de Educación Superiorreúne representantes de las universidades públicas y privadas, asociaciones y redes académicas, sindicatos docentes, organizaciones estudiantiles y gobiernos de los países de la región, apoyada y auspiciada por la UNESCO mediante el Instituto de Educación Superior de América Latina y el Caribe (IESALC).

3 La ley 24521 de Educación Superior, en vigencia desde el año 1995, establece a partir de estas modificaciones la responsabilidad "indelegable y principal” del Estado respecto de la educación superior, considerándola un "bien público" y un "derecho humano".

4 Es necesario mencionar que el egreso en el marco del Departamento de Sociología ha sido estudiado en distintos trabajos publicados previamente, en los que se han analizado los perfiles de los/as graduados/as de la Licenciatura (Giordana, Erbetta y Manuele, 2003) y las características de los trabajos finales o tesinas (Alzugaray, Cafiero y Varela, 2012). A su vez, se han reconstruido y analizado las trayectorias universitarias de los/as estudiantes de las carreras de Sociología (Cotignola, Legarralde y Margueliche, 2017) y también sus trayectorias laborales y educativas (Di Bello, Fernández Berdaguer y Santos, 2011).

5 Se trata de materias avanzadas y especializadas, que suponen un paso por las materias troncales de las carreras. De acuerdo con el Plan de la Carrera (aprobado en 2001 e implementado a partir de 2003), las optativas C, D y E tienen contenidos de teoría social o sociología especial. En este sentido, respectivamente, refieren al desarrollo de contenidos teóricos, autores o corrientes analíticas relevantes para el estudio de la sociedad o abordan problemas específicos mediante herramientas analíticas, metodológicas y técnicas propias del saber sociológico. La Teoría Social Contemporánea B, en complementación con Teoría Social Contemporánea A, refiere a materias que ofrecen una panorámica amplia de las principales tradiciones y corrientes analíticas, y autores, del pensamiento social contemporáneo. Por último, los talleres de investigación tienen como objetivo la adquisición y desarrollo de aptitudes y habilidades necesarias para abordar el diseño y realización de proyectos de investigación, en las diversas modalidades y técnicas que son propias de la investigación sociológica.

6 De hecho, esta es la idea que anima el Plan de Estudios de la Licenciatura. Este objetivo en el papel, no obstante, presenta algunas dificultades para su efectivización en la dinámica concreta de las materias. 